\title{
The Relationship between Cell Phone Use, Physical Activity, and Sedentary Behavior in United States Adults above College-age
}

\author{
Curtis Fennell $^{1, *}$, Ellen L. Glickman ${ }^{2}$, Andrew Lepp ${ }^{2}$, J. Derek Kingsley ${ }^{2}$, Jacob E. Barkley ${ }^{2}$ \\ ${ }^{1}$ Exercise and Nutrition Science, University of Montevallo, Alabama, United States \\ ${ }^{2}$ Exercise Physiology, Kent State University, Ohio, United States
}

Copyright $\bigcirc 2018$ by authors, all rights reserved. Authors agree that this article remains permanently open access under the terms of the Creative Commons Attribution License 4.0 International License

\begin{abstract}
Purpose: There is evidence of a positive relationship between cellular telephone use and sedentary behavior but not physical activity in college-aged individuals (18-29 years). These relationships have not been tested in individuals older than college age $(\geq 30$ years old). Testing these relationships in older individuals is warranted as cell phone use is inversely associated with age. Methods: A sample of adults aged 30-63 years $(\mathrm{N}=69$, $50.5 \pm 8.2$ years old) wore a physical activity monitor (accelerometer) for seven days and completed validated surveys assessing daily cell phone use, physical activity, and sedentary behavior. Results: Cell phone use $(\bar{x}=$ $125.2 \pm 146.8$ minutes per day) was inversely associated with age $(r=-0.3, p=0.005)$. Cell use was not associated with objectively- or subjectively-measured physical activity or sedentary behavior $(\mathrm{r} \leq 0.1, \mathrm{p} \geq 0.3)$. Tertile splits were performed to establish groups of low, moderate, and high cell phone users. There were no significant $(\mathrm{F} \leq$ $2.0, \mathrm{p} \geq 0.12$ for all) differences in physical activity or sedentary behavior between groups. Conclusion: Unlike what has been reported in college-aged individuals, cell use was not associated with sedentary behavior in adults older than college age.
\end{abstract}

Keywords Behavioral Science, Health, Health Promotion, Technology

\section{Introduction}

Internet-connected, mobile, cellular telephone (henceforth cell phone) use has become increasingly common in the last decade. According to the most recent data, $91 \%$ of United States adults report owning a cell phone with $64 \%$ possessing internet-connected cell phones (i.e., smartphones) which allow for access to a wide array of screen-based activities including, but not limited to: streaming videos, searching the internet, playing video games and updating social media sites [1]. These screen-based activities have traditionally been considered sedentary pursuits [2, 3]. However, because modern smartphones are portable and provide the user access to physical activity monitoring software, software applications (i.e., "apps") designed to promote physical activity, and because these devices can be used effectively as part of interventions to prompt participants to increase physical activity (i.e., mobile health or mHealth), smartphone use does not have to be a sedentary activity [4-11].

Excessive sedentary (i.e., sitting) behavior and physical inactivity are of concern as both are independently associated with an increased risk of a multitude of health problems including the metabolic syndrome, cardiovascular disease, and type 2 diabetes [12-14]. Currently an estimated 3.2 million people around the world may die each year due to the negative health effects related to excessive sedentary behavior [15]. Conversely, physical activity is associated with many positive health effects including improved functional and cognitive health, reduced risk of falls, prevention of cardiovascular disease/coronary artery disease, hypertension, stroke, osteoporosis, type 2 diabetes mellitus, metabolic syndrome, obesity, colon cancer, breast cancer, and depression, and a decreased risk of premature mortality [16-18]. Despite this widely available information, many adults fail to participate in adequate physical activity and are excessively sedentary [16, 19-22].

Because many adults are overly sedentary and participate in inadequate amounts of physical activity, an understanding of factors which contribute to sedentary behavior and physical inactivity may provide targets for interventions designed for disease prevention and control as well as public health awareness. Much of adults' sedentary time is allocated to electronic screen use, such as 
watching television or using a computer $[12,23]$. The modern smartphone provides users with access to these historically sedentary behaviors, in virtually any setting and at any time. While certain smartphone functions (e.g., activity monitors, fitness "apps") have the potential to promote physical activity, there is evidence in college-aged individuals that total cell phone use is positively associated with sedentary behavior, negatively related to cardiorespiratory fitness, and may reduce intensity if used during exercise or free-living walking for active transport [24-28]. These prior studies on college students indicated that high cell phone users engaged in significantly more (78-145 more minutes per day) sedentary behavior per day than low and moderate use peers $[25,27,28]$. These findings support the notion of the modern smartphone as a sedentary device in college-aged adults. However, while these previous studies report that cell phone use was related to sedentary behavior and may interfere with exercise, resulting in reduced intensity, there is no evidence of a link between cell phone use and total physical activity behavior. Furthermore, these assessments are limited to only college students.

While these previous reports outline the potential of the modern smartphone as a sedentary device in college students, there is evidence of an inverse relationship between cell phone use and age $[1,25]$. Considering this, it is then possible that examining older adults (i.e., $\geq 30$ years old) may yield different outcomes than these previous findings. Additionally, these previous studies are limited to using subjective measures to assess physical activity (i.e., surveys) which may be inferior to objective measures (e.g., physical activity monitors or accelerometers) [22, 29-31]. Therefore, the purpose of this investigation was to assess the relationship between cell phone use, physical activity, and sedentary behavior using validated objective physical activity monitors and subjective measurements in adults beyond college age ( $\geq 30$ years old). Because previous research with college-aged individuals found cell phone use to be positively associated with sedentary behavior and not related to physical activity, our hypothesis was there would be a similar positive relationship between cell phone use and sedentary behavior, but not physical activity in the current sample.

\section{Methodology}

\subsection{Participants}

Data collection and analysis occurred from December 2016 to May 2017.

A sample of 69 employees $(51 \pm 8$ years old, $n=54$ females) from a large, public university in the Midwestern United States were recruited to participate in the current investigation via advertisement through e-mail and a website. Of these participants, 53 ( $n=49$ females) were recruited from individuals participating in a university faculty and staff exercise intervention program, which was open to all employees at the university. The remaining 16 participants ( $n=11$ females) were individuals engaging in a control group for the above intervention. The data collection for the current investigation took place at the beginning (first week) of this exercise intervention. The exercise intervention consisted of group exercise classes which were offered three times per week where participants could freely attend as few or as many sessions as desired. Both the intervention and control groups completed fitness testing for this intervention.

Prior to data collection, all participants were familiarized with the protocol, including instruction on the benefits and risks of the study, and provided written consent. Medical history forms were completed prior to participation. Participants were excluded if they reported a history of medical disorders for issues such as orthopedic injuries, cardiovascular disorder, etc. that would preclude them for participating in regular physical activity. All study procedures were approved by the university Institutional Review Board.

\subsection{Procedures}

The participants reported to the exercise physiology laboratory on two separate occasions, separated by one week. During the first visit, participants were given a validated accelerometer (Movband, Movable, Cleveland, $\mathrm{OH})$ to wear around the wrist of their dominant hand as much as possible (a minimum of $\geq 10$ hours per day) during waking hours for a one week period [32]. A questionnaire assessing age (years) and sex (male, female) was also completed by each participant during this first visit. Additionally, during this first visit, participants were given an activity monitor report to complete each night for one week, which assesses when the Movband accelerometer was worn each day. Participants' data would have been excluded if they did not wear the accelerometer for a minimum of $\geq 10$ hours per day. However, all of the participants successfully wore the monitors for the requisite amount of time. The second visit occurred one week after the first visit, which consisted of participants returning to the laboratory to complete self-reported physical activity and sedentary behavior measures using the International Physical Activity Questionnaire (IPAQ) [33]. Additionally, during the second visit, participants self-reported their cell phone use via a validated survey for each day of the previous week [28, 34, 35]. Finally, the participant's Movband accelerometer data from the previous week were uploaded and processed using software provided by the manufacturer.

\subsection{Measures}

Physical activity behavior was objectively measured via the Movband accelerometer, which has been validated 
against a previously-validated, research-grade accelerometer (Actigraph GT1M, Actigraph Corporation, Pensacola, FL) and indirect calorimetry [32]. The Movband is a three-plane accelerometer that measures acceleration to quantify an accurate estimate of physical activity. Physical activity is recorded as "moves" and a built-in algorithm is used to convert the movement data from "moves" to steps and miles, which were used as the measures herein because these units are more widely understood. The data from the Movbands are downloadable with free computer software provided by the manufacturer http://www.movable.com/ (last accessed $6 / 24 / 17$ ). All participants were instructed to wear the Movband a minimum of $\geq 10$ hours per day for seven days during waking hours. Participants were asked to complete a daily self-reported questionnaire which assesses how often they wore the Movband. This was done to quantify the amount of minutes the participant wore the Movband. That information was then used to calculate miles per minute (miles per minute $=$ total miles $\div$ time worn) and steps per minute (steps per minute $=$ total steps $\div$ time worn).

The IPAQ instrument is a validated self-reported measure of physical activity and sedentary behavior. The IPAQ has been validated in studies carried out by a number of countries [36]. Physical activity is assessed as behaviors performed for at least 10 minutes at three intensities: walking (low intensity), moderate, and vigorous. Users are given a "Continuous score" (Metabolic Equivalent of Task for the week) based on amount of time in each intensity and a "Categorical score" (1-3 scale), which determines if the user is inactive, minimally active, or health enhancing physically active. The measures of physical activity are then summed to estimate the total amount of time spent participating in physical activity per week. Total daily physical activity is expressed as Metabolic Equivalent of Task-minutes per day (MET-min per day). Sedentary time was recorded as the sum of minutes sitting during the five weekdays and two weekend days.

The total daily cell phone use was assessed as follows: "As accurately as possible, please estimate the total amount of time you spend using your mobile phone each day. Please consider all uses except listening to music. For example, consider calling, texting, Facebook, e-mail, sending photos, gaming, surfing the Internet, watching videos, and all other uses driven by 'apps' and software" $[28,34,35]$. This questionnaire is a valid predictor of objectively measured cell phone use and is similar to other survey devices designed to assess electronic media consumption (e.g., watching television) [37].

\subsection{Statistical Analysis}

Statistical Packages for Social Sciences version 21.0 was used to analyze the data. Significance was set $a$ priori at $\alpha$ $\leq 0.05$. An independent samples t-test was conducted to determine any differences in cell phone use, physical activity, and sedentary behavior between the aforementioned exercise program intervention and control groups from which the participants in the current investigation also participated. Pearson's correlation analyses were performed to assess the relationship between cell phone use and the following variables: sex, age, weekly physical activity in MET-min per week, walking MET-min per week, moderate physical activity MET- min per week, vigorous physical activity MET-min per week, weekly minutes of sitting, and miles per minute and steps per minute as measured via the Movband. To further assess potential relationships between cell phone use and the aforementioned dependent variables, participants were split into tertiles based on total cell phone use: low use $(<$ 33rd percentile, $23.1 \pm 16.9$ min of use per day, $n=21$ ), moderate use (33rd to 66 th percentile, $89.0 \pm 27.8 \mathrm{~min}$ of use per day, $n=29$ ), and high use ( $>66$ th percentile, 295.3 $\pm 190.3 \mathrm{~min}$ of use per day, $n=19$ ) and a multivariate analysis of covariance (MANCOVA) was conducted to determine if there were differences across the three cell phone use groups (low, moderate, high) in miles per minute, steps per minute, MET-min per week, and sedentary behavior. Sex and age were included as covariates because age was significantly related to cell phone use in the current analysis and prior research has shown both factors to be correlates of cell phone use and physical activity [1, $38,39]$. This analysis is similar to previous methods employed by Barkley et al., (2015) that assessed the relationship between sedentary behavior and cell phone use in college-aged individuals. These tertiles allowed us to quantify the miles per minute, steps per minute, MET min per week, and minutes of sedentary behavior in which high cell phone users participated in versus that of their lower use peers. Additionally, to further examine the potential effects of age on cell phone use, physical activity, and sedentary behavior, a separate tertile split was conducted and participants were grouped as follows: younger age $(<$ 33 rd percentile, $170.0 \pm 29.7 \mathrm{~min}$ of use per day, $n=26$ ), middle age (33rd to 66th percentile, $123.5 \pm 31.7 \mathrm{~min}$ of use per day, $n=24$ ), and older age ( $>$ 66th percentile, 60.3 \pm 36.0 min of use per day, $n=19$ ). A MANCOVA was conducted to determine if there were differences across the three age groups (younger, middle, and older) in cell phone use, miles per minute, steps per minute, MET min per week, and sedentary behavior. Sex was included as a covariate.

\section{Results}

\subsection{Differences between the Intervention and Control Groups}

The independent samples t-test revealed there were no significant differences between the intervention and control groups cell phone use $(\mathrm{t}=0.2, p=0.7)$, sedentary behavior $(\mathrm{t}=1.2, p=0.5)$, subjective physical activity $(\mathrm{t}=$ 
$0.6, p=0.3)$, and objective physical activity $(\mathrm{t}=0.7, p=$ $0.3)$.

\subsection{Physical Characteristics and Cell Phone Use}

Descriptive statistics are provided in table 1. Mean cell phone use was $125.2 \pm 146.8$ minutes per day. Cell phone use was inversely $(r=-0.3, p=0.005)$ associated with age. In other words, younger participants reported greater total cell phone usage. Cell phone use was not different $(r=-0.1$, $p=0.5$ ) between males (dummy coded as 0 ) and females (dummy coded as 1).

Table 1. Participants Descriptive Statistics

\begin{tabular}{cc}
\hline Variable & Mean \pm SD \\
\hline Participants & $N=69(15$ male, 54 \\
Age $(\mathrm{yrs})$ & $51 \pm 8$ \\
Height $(\mathrm{cm})$ & $166.5 \pm 8.8$ \\
Weight $(\mathrm{kg})$ & $83.4 \pm 23.3$ \\
BMI $\left(\mathrm{kg} / \mathrm{m}^{2}\right)$ & $30.0 \pm 7.7$ \\
Miles per minute (miles) & $0.00618 \pm 0.00263$ \\
Steps per minute (steps) & $11.5 \pm 4.2$ \\
IPAQ MET-min per week (METS) & $1501.9 \pm 2048.7$ \\
IPAQ Categorical Score (units) & $1.6 \pm 0.7$ \\
IPAQ Walking (min) & 178.8 \\
IPAQ Moderate (min) & 89.6 \\
IPAQ Vigorous (min) & 78.8 \\
Sedentary time per day (min) & $431 \pm 181$ \\
Total Cell Phone use per day (min) & $125.2 \pm 146.8$ \\
\hline
\end{tabular}

\subsection{Cell Phone use, Physical Activity and Sedentary Behavior}

There were no relationships between cell phone use and physical activity, including Movband miles per minute $(\mathrm{r}=$ $0.1, p=0.3)$, Movband steps per minute $(\mathrm{r}=0.06, p=0.6)$, IPAQ MET min per week $(\mathrm{r}=0.1, p=0.3)$, IPAQ Categorical score $(\mathrm{r}=0.06, p=0.6)$, IPAQ Walking $(\mathrm{r}=$ $0.1, \mathrm{p}=0.4)$, IPAQ Moderate $(\mathrm{r}=-0.07, p=0.6)$, and IPAQ Vigorous $(\mathrm{r}=0.06, p=0.6)$. Additionally, total cell phone use was not significantly related to sedentary behavior $(\mathrm{r}=-0.11, p=0.4)$, (table 1$)$.

\subsection{Cell Phone Use Groups}

There were no significant differences $(F \leq 1.0, p \geq 0.4$ for all) in objectively measured physical activity measured in miles per minute or subjective measures of physical activity measured as MET-min per week, and sedentary behavior across the three cell phone use groups (low, moderate, and high cell phone users) (table 2, figures 1 and 2). There were significant differences $(F=5.3, p=0.007)$ for steps per minute such that there were significantly greater $(t=2.5, p=0.015)$ steps per minute in the low users $(12.5 \pm 4.8)$ than the moderate users $(9.7 \pm 3.1)$ and significantly greater $(t=3.3, p=0.002)$ steps per minute in the high users $(13.2 \pm 4.3)$ than the moderate users but no differences $(t=0.5, p=0.6)$ of steps per minute between the low and high users, (figure 3 ).

Table 2. Cell Phone Use Tertile Groups (Data are Mean \pm SEM)

\begin{tabular}{cccc}
\hline & $\begin{array}{c}\text { Low Cell } \\
\text { Phone Use } \\
(<\mathbf{3 3 \%})\end{array}$ & $\begin{array}{c}\text { Moderate } \\
\text { Cell Phone } \\
\text { Use } \\
(\mathbf{3 3 - 6 6 \% )}\end{array}$ & $\begin{array}{c}\text { High Cell } \\
\text { Phone Use } \\
(>\mathbf{6 6 \%})\end{array}$ \\
\hline Cell Phone Use & $n=21,23.1$ & $n=29,89.0 \pm$ & $n=19$, \\
(min per day) & \pm 16.9 & 27.8 & $295.3 \pm$ \\
& & & 190.3 \\
\hline
\end{tabular}

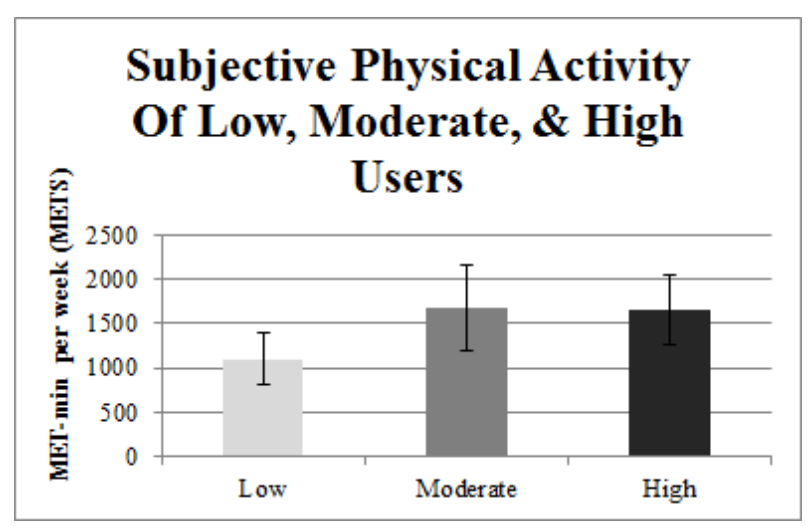

Figure 1. Subjective physical activity among low, moderate, and high cell phone users $($ Mean \pm SEM)

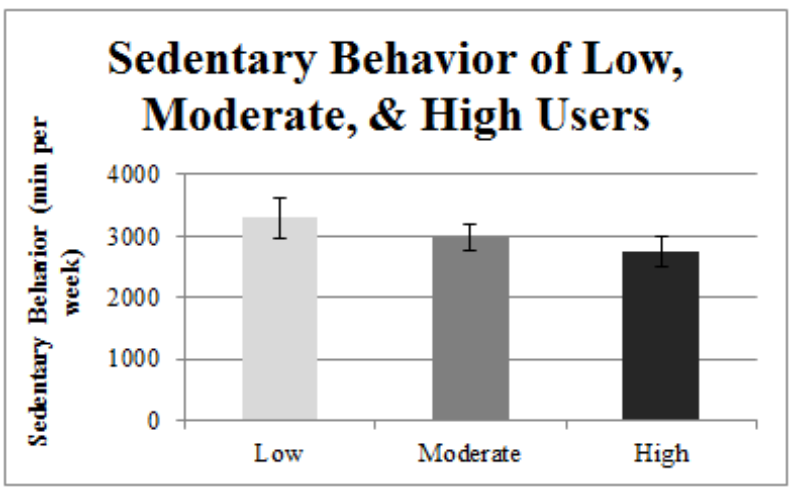

Figure 2. Sedentary behavior among low, moderate, and high cell phone users (Mean $\pm \mathrm{SEM}$ )

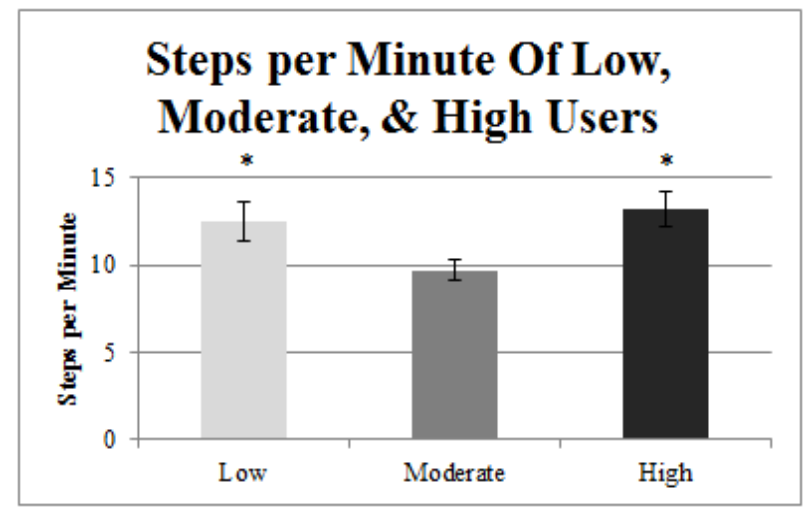

Figure 3. Steps per minute of low, moderate, and high cell phone users $(\mathrm{Mean} \pm \mathrm{SEM})$

*Significant difference from moderate users $p \leq 0.05$ 


\subsection{Age Groups}

There were no significant differences for any measure of physical activity $(F \leq 1.1, p \geq 0.4$ for all) or sedentary behavior $(F=1.0, p=0.6)$ across the three age groups $(n=$ 26 younger group, $n=24$ middle group, $n=19$ older group). Additionally, there was not a significant difference $(F=2.8$, $p=0.07$ ) for the three age groups' cell phone use, although this was trending significance (table 3 ).

Table 3. Age Tertile Groups for Cell Phone Use (Data are Mean \pm SEM)

\begin{tabular}{cccc}
\hline & $\begin{array}{c}\text { Younger } \\
\text { Age }(<\mathbf{4 9} \\
\text { years old })\end{array}$ & $\begin{array}{c}\text { Middle Age } \\
(\mathbf{4 9 - 5 6} \text { years } \\
\text { old })\end{array}$ & $\begin{array}{c}\text { Older Age } \\
(<\mathbf{5 6} \text { years } \\
\text { old })\end{array}$ \\
\hline $\begin{array}{c}\text { Cell Phone Use } \\
\text { (min per day) }\end{array}$ & $n=26$, & $n=24,123.5$ & $n=19,60.3$ \\
& $170.0 \pm 29.7$ & \pm 31.7 & \pm 36.0 \\
\hline
\end{tabular}

\section{Discussion}

The present investigation assessed the relationship between cell phone use, age, physical activity, and sedentary behavior in adults ( $51 \pm 8$ years old) greater than college age. This is the first study we are aware of which examined the relationship between cell phone use and physical activity and sedentary behavior, in adults greater than college age (i.e. $\geq 30$ years old). This was also the first study we are aware of which examined the relationship between cell phone use and objectively-measured physical activity. There was an inverse relationship between cell phone use and age. There was also a trend towards a main effect of age group for differences in cell phone use with younger participants using their device for 47 and 110 more minutes per day than middle- and older-age participants, respectively. No relationships existed between the measures of cell phone use and physical activity or sedentary behavior.

Presently cell phone use was not associated with physical activity in adults 30-65 years old. This is consistent with previous results in college-aged individuals $[27,28]$. However, the lack of a relationship between cell use and sedentary behavior is different from previous research in college students. Previously, cell phone use in college-aged individuals (18-29 years old), was positively associated with sedentary behavior, and high cell phone users participated in significantly more (495-584 minutes per day) sedentary behavior, compared to moderate (417-491 minutes per day) and low (395-439 minutes per day) users $[25,27]$. The reason for these disparate findings of the present study and those previous may be differences in how college-age adults and adults beyond college age use their cell phone and select their sedentary activities.

There was an inverse relationship between age and cell phone use in the present study which is consistent with previous research $[25,40]$. The presented means from the tertile split by age indicates the younger age group participated in $38 \%$ and $180 \%$ greater daily cell phone use than the middle- and older-age groups, respectively. Interestingly, when comparing previous data on college-aged individuals (18-29 years old) with the present sample of adults (30-63 years old), the average cell phone use is drastically different. College-aged individuals have reported using the cell phone for means ranging from 300 to 380 minutes per day $[25,27,28]$, which is much greater than the mean use in the present sample of 125 minutes per day. The difference between the two age groups' cell phone use and sedentary behavior may stem from generational differences; the younger generation is growing up in a digital age which allows constant connection to digital activities [41], where cell phone use has become the hub of a digital lifestyle [42]. These younger, "digital natives" may function as being constantly connected to people and information through their mobile cellular devices [43]. As such, the cell phone may have become the sedentary screen of choice in college-aged individuals and if they are participating in sedentary behavior their cell phone is likely to be incorporated into that behavior. Conversely, the above college-age population may be engaging in more traditional forms of sedentary behaviors, such as watching television or videos, reading, and using a desktop or laptop computer. A study showed United States adults spend 7-9 hours of their work day sedentary [44] and the greatest sedentary time activity consisted of television viewing and screen time [23]. Hence, cell phone use may not be a large contributor to sedentary behavior in individuals above the college-age but it is possible that low cell phone users beyond college age could still be highly sedentary since they may prefer these traditional sedentary behaviors.

Although this investigation provides novel information regarding the relationship between modern cell phone use with physical activity and sedentary behavior in adults older than college age it is not without limitations. Since this was a non-experimental study, causal inference cannot be made. A second limitation is the context in which participants were using their cell phones was not captured (e.g. were participants using their cell phones while they were sitting). Additionally, both cell phone use and the IPAQ sedentary assessment are self-report measures, and therefore are subjective in nature. Experimental research designs which capture the individual's physical activity and sedentary behavior during cell phone use (e.g. when the cell phone is used does one sit, stand, walk) utilizing objective measures of cell phone use and questions regarding the context of cell phone use would fulfill these two limitations and are suggested for future research. A third limitation is the fact that this was a relatively small sample size of individuals from the ages of 30-63, at a single, large, public university in the Midwestern United States. A sample size of 69 was chosen because this study used objective and subjective measures of physical activity versus using an easier assessment such as only subjective measures of physical activity which were the measurement choice used in previous studies assessing similar variables 
[24-28]. Objective measures of physical activity were used in the current investigation because objective measures may be superior to subjective measures [22, 29-31, 45, 46]. Additionally, only $21 \%$ of the participants were male, which limits the ability to generalize the results of this study. Furthermore, the participants included individuals who were either involved in a university exercise program intervention or in a control group of the intervention. Because there were no statistical differences between the groups physical activity, sedentary behavior, or cell phone use, this sample may be representative of the general adult population. Since all participants in this investigation wore an objective physical activity monitor with visual feedback for the user, this may also be a limitation because visual feedback may have a positive effect on one's physical activity and sedentary behavior, by creating awareness and the role of feedback may reinforce motivation to engage in a behavior [47, 48]. Hence, future research should measure larger samples or different populations including: various ethnic groups, different types of jobs, different regions, a greater proportion of males, and use objective physical activity monitors which do not provide visual feedback to the user, which may yield differing results than this report.

\section{Conclusions}

In conclusion, this was the first study we are aware of to assess the relationship between cell phone use and physical activity and sedentary behavior in adults greater than the college age. This was also the first examination of these relationships using both subjective and objective measures of physical activity in any age group. Cell phone use was inversely associated with age, as has been shown previously. However, cell phone use was not related to physical activity or sedentary behavior. This is different from a previously-demonstrated positive relationship between cell phone use and sedentary behavior in 18-29 year-old college students. Because younger individuals are "digital natives" who have been raised with near-constant access to cell phones this may be their sedentary activity of choice. Conversely, adults who are older may prefer other, more traditional forms of sedentary activities such as watching television and using a desktop computer. This is important information because much of adults' sedentary time is allocated to electronic screen use and the modern smartphone provides access to screen use but also has features which enable it to promote physical activity. Understanding if the smartphone contributes to sedentary behavior and physical inactivity across all ages may provide focus areas for interventions designed for health promotion and public health awareness messages.

\section{Acknowledgements}

There are no acknowledgments.

\section{Compliance with Ethical Standards}

Funding: There was no funding for this research investigation.

Conflict of Interest: There are no conflicts of interest for this study.

Ethical Approval: All procedures performed were in accordance with the ethical standards of the human subjects institutional review board.

Informed Consent: Informed consent was obtained from all individual participants included in the study.

\section{REFERENCES}

[1] Zickuhr K. Generations and their gadgets. Pew Internet \& American Life Project. 2011;20.

[2] Rosenberg DE, Norman GJ, Wagner N, et al. Reliability and validity of the sedentary behavior questionnaire (SBQ) for adults. J Phys Act Health. 2010;7(6):697-705.

[3] King A, Goldberg J, Salmon J. Correlates of prolonged television viewing time in US adults to inform program development. Am J Prev Med. 2010;38(1):17-26.

[4] Fjeldsoe BS, Miller YD, Marshall AL. MobileMums: a randomized controlled trial of an SMS-based physical activity intervention. Annals Behav Med. 2010;39(2):101-111.

[5] Kwapisz JR, Weiss GM, Moore SA. Activity recognition using cell phone accelerometers. ACM SigKDD Explorations Newsletter. 2011;12(2):74-82.

[6] Toscos T, Faber A, Connelly K, et al. Encouraging physical activity in teens Can technology help reduce barriers to physical activity in adolescent girls? Proceedings of Pervasive Health 2008:218-221.

[7] Consolvo S, Klasnja P, McDonald DW, et al. Flowers or a robot army?: encouraging awareness \& activity with personal, mobile displays. Proceedings of UbiComp: ACM:54-63.

[8] Consolvo S, McDonald DW, Toscos T, et al. Activity sensing in the wild: a field trial of ubifit garden. Proceedings of the SIGCHI Conference on Human Factors in Computing Systems: ACM 2008:1797-1806.

[9] Lyons EJ, Lewis ZH, Mayrsohn BG, et al. Behavior change techniques implemented in electronic lifestyle activity monitors: a systematic content analysis. J Med Int Res. 2014;16(8).

[10] Hurling R, Catt M, De Boni M, et al. Using internet and mobile phone technology to deliver an automated physical activity program: randomized controlled trial. J Med Int Res. 2007;9(2):e7.

[11] King A, Hekler E, Grieco L, et al. Harnessing different motivational frames via mobile phones to promote daily physical activity and reduce sedentary behavior in aging adults. PloS One. 2013;8(4):e62613. 
[12] Ford ES, Kohl HW, Mokdad AH, et al. Sedentary behavior, physical activity, and the metabolic syndrome among US adults. Obes Res. 2005;13(3):608-614.

[13] Lee I-M, Shiroma EJ, Lobelo F, et al. Effect of physical inactivity on major non-communicable diseases worldwide: an analysis of burden of disease and life expectancy. The Lancet. 2012;380(9838):219-229.

[14] Owen N, Healy GN, Matthews CE, et al. Too much sitting: the population-health science of sedentary behavior. Exerc Sport Sci Rev. 2010;38(3):105.

[15] Pratt M, Norris J, Lobelo F, et al. The cost of physical inactivity: moving into the 21st century. British J Sport Med. 2014;48(3):171-173.

[16] Garber CE, Blissmer B, Deschenes MR, et al. American College of Sports Medicine position stand. Quantity and quality of exercise for developing and maintaining cardiorespiratory, musculoskeletal, and neuromotor fitness in apparently healthy adults: guidance for prescribing exercise. Med Sci Sports Exerc. 2011;43(7):1334-1359.

[17] Physical-Activity-Guidelines-Committee. Physical activity guidelines advisory committee report, 2008. Washington, DC: US Department of Health and Human Services 2008:A1-H14.

[18] Vogel T, Brechat PH, Leprêtre PM, et al. Health benefits of physical activity in older patients: a review. Int J Clin Prac. 2009;63(2):303-320.

[19] American-College-Health-Association. National College Health Assessment II: Undergraduate Reference Group Executive Summary Spring 2012. Hanover, MD: Am Coll Health Assoc. 2012;60(8):556-561.

[20] Haskell WL, Lee I-M, Pate RR, et al. Physical activity and public health: updated recommendation for adults from the American College of Sports Medicine and the American Heart Association. Circulation. 2007;116(9):1081-1096.

[21] Troiano RP, Berrigan D, Dodd KW, et al. Physical activity in the United States measured by accelerometer. Med Sci Sports Exerc. 2008;40(1):181-188.

[22] Centers-for-Disease-Control-Prevention. Adult participation in aerobic and muscle-strengthening physical activities--United States, 2011. Morb Mort Wkly Rep. 2013;62(17):326-330.

[23] Mansoubi M, Pearson N, Biddle SJ, et al. The relationship between sedentary behaviour and physical activity in adults: A systematic review. Prev Med. 2014;69:28-35.

[24] Rebold MJ, Lepp A, Sanders GJ, et al. The impact of cell phone use on the intensity and liking of a bout of treadmill exercise. Plos One. 2015;10(5):1-12.

[25] Barkley JE, Lepp A. Mobile phone use among college students is a sedentary leisure behavior which may interfere with exercise. Comp Hum Behav. 2016;56:29-33

[26] Barkley JE, Lepp A. Cellular telephone use during free-living walking significantly reduces average walking speed. BMC research notes. 2016;9(1):1.

[27] Barkley JE, Lepp A, Salehi-Esfahani S. College students' mobile telephone use is positively associated with sedentary behavior. Am J Lifestyle Med. 2015; doi: $10.1177 / 1559827615594338$.

[28] Lepp A, Barkley JE, Sanders GJ, et al. The relationship between cell phone use, physical and sedentary activity, and cardiorespiratory fitness in a sample of U.S. college students. Int J Behav Nutr Phys Act. 2013;10:79-89.

[29] Tucker JM, Welk GJ, Beyler NK. Physical activity in US adults: compliance with the physical activity guidelines for Americans. Am J Prev Med. 2011;40(4):454-461.

[30] Shephard RJ. Limits to the measurement of habitual physical activity by questionnaires. British J Sports Med. 2003;37(3):197-206.

[31] Leenders NY, Sherman WM, Nagaraja H. Comparisons of four methods of estimating physical activity in adult women. Med Sci Sports Exerc. 2000;32(7):1320-1326.

[32] Barkley JE, Rebold M, Carnes A, et al. The validity of a commercially-available, low-cost, wrist-mounted accelerometer during treadmill exercise Med Sci Sports Exerc. 2014;46(5):490.

[33] Booth ML, Ainsworth BE, Pratt M, et al. International physical activity questionnaire: 12-country reliability and validity. Med Sci Sports Exerc. 2003;35(8):1381-1395.

[34] Lepp A, Barkley JE, Karpinski AC. The relationship between cell phone use, academic performance, anxiety, and satisfaction with life in college students. Comp Hum Behav. 2014;31:343-350

[35] Lepp A, Barkley JE, Karpinski AC. The relationship between cell phone use and academic performance in a sample of US college students. SAGE Open. 2015;5(1):1-9.

[36] Craig C, Marshall A, Sjöström M, et al. Consensus Group and the IPAQ Reliability and Validity Study Group. International Physical Activity Questionnaire (IPAQ): 12-country reliability and validity. Med Sci Sports Exerc. 2003;35(8):1381-1395.

[37] Clark BK, Sugiyama T, Healy GN, et al. Validity and reliability of measures of television viewing time and other non-occupational sedentary behaviour of adults: a review. Obes Rev. 2009;10(1):7-16.

[38] Sánchez-Martínez M, Otero A. Factors associated with cell phone use in adolescents in the community of Madrid (Spain). CyberPsych Behav. 2009;12(2):131-137.

[39] Caspersen CJ, Pereira MA, Curran KM. Changes in physical activity patterns in the United States, by sex and cross-sectional age. Med Sci Sport Exerc. 2000;32(9):1601-1609.

[40] Pew-Research-Center. Pew Research Center Internet Project Survey. Washington, DC 2014. 2014.

[41] Anderson J, Rainie L. Millennials will benefit and suffer due to their hyperconnected lives. Washington DC, Pew Research Center. 2012.

[42] Bajarin T. Your Smartphone Will Become the Hub of Your Digital Lifestyle. Time. 2013.

[43] Palfrey J, Gasser U. Born digital: Understanding the first generation of digital natives: Basic Books 2013. 
[44] Matthews CE, Chen KY, Freedson PS, et al. Amount of time spent in sedentary behaviors in the United States, 20032004. Am J Epidimiology. 2008;167(7):875-881.

[45] Sallis JF, Saelens BE. Assessment of physical activity by self-report: status, limitations, and future directions. Res Quart Exerc Sport. 2000;71(2):1-14.

[46] Seefeldt V, Malina RM, Clark MA. Factors affecting levels of physical activity in adults. Sports Med.
2002;32(3):143-168.

[47] Patterson R. Changing patient behavior: Improving outcomes in health and disease management: Jossey-Bass Inc Pub 2001.

[48] Proper K, Van der Beek A, Hildebrandt V, et al. Short term effect of feedback on fitness and health measurements on self reported appraisal of the stage of change. Brit J Sports Med. 2003;37(6):529-534. 